\title{
Interlaboratory variability of MIB1 staining in well-differentiated pancreatic neuroendocrine tumors
}

\author{
Annika Blank ${ }^{1} \cdot$ Laura Wehweck $^{2}$ - Ilaria Marinoni ${ }^{1}$ - Laura Amanda Boos ${ }^{1}$. \\ Frank Bergmann ${ }^{3}$ - Anja Maria Schmitt ${ }^{1}$ - Aurel Perren ${ }^{1}$
}

Received: 6 February 2015 / Revised: 16 July 2015 / Accepted: 23 August 2015 / Published online: 17 September 2015

(C) Springer-Verlag Berlin Heidelberg 2015

\begin{abstract}
Neuroendocrine tumors (NET) are routinely graded and staged to judge prognosis. Proliferation index using MIB1 staining has been introduced to assess grading. There are vivid discussions on cutoff definitions, automated counting, and interobserver variability. However, no data exist regarding interlaboratory reproducibility for low proliferation indices which are of importance to discriminate between G1 and G2 NET. We performed MIB1 staining in three different university hospital-based pathology laboratories on a tissue micro array (TMA) of a well-characterized patient cohort, containing pancreatic NET of 61 patients. To calculate the proliferation index, number of positive tumor nuclei was divided by the total number of tumor nuclei. Labeling index was compared to mitotic counts in whole tissue sections and to clinical outcome. Linear regression analysis, intraclass comparison, and log-rank analysis were performed. Intraclass correlation showed moderate-to-fair agreement. Especially low proliferating tumors were affected by interlaboratory differences. Log-rank analysis was performed for each lab and resulted in three different cutoffs $(5.0,3.0$, and $0.5 \%$ ). Every calculated cutoff stratified the patient cohort to a significant extent for the underlying stain $(p<0.001,<0.001$, and $<0.001)$ but showed no or lesser significance when applied to the other
\end{abstract}

Annika Blank

annika.blank@pathology.unibe.ch

1 University of Bern, Institute of Pathology, Murtenstr. 31, CH-3012 Bern, Switzerland

2 Department of Cardiology, Pneumology and Internal Intensive Care Medicine, Klinikum Neuperlach, Städtisches Klinikum München GmbH, Oskar-Maria-Graf-Ring 5, 83575 Munich, Germany

3 University of Heidelberg, Institute of Pathology, Im Neuenheimer Feld 224, 69120 Heidelberg, Germany stains. Significant and relevant interlab differences for MIB1 exist. Since the MIB1 proliferation index influences grading, local cutoffs or external standardization should urgently be introduced to achieve reliability and reproducibility.

Keywords Interlaboratory variability $\cdot \mathrm{MIB} 1 \cdot \mathrm{Ki} 67$. Neuroendocrine $\cdot$ Grading

\section{Introduction}

Pancreatic neuroendocrine tumors (pNET) have a wide range of biological behavior which is difficult to predict [1-4]. Metastasis is the most important predictor of survival, but also among metastasized tumors, the survival time is still very variable [2, 5-7]. In the absence of metastases, prediction of relapse is also challenging. To date, pNET are stratified mainly according to stage and grade. Grading has been introduced as an important parameter by the WHO 2004 classification [8] and has formally been proposed by the European Neuroendocrine Tumor Society (ENETS) [6]. Since its proposal in 2006, grading has been implicated in decision-making and in choosing treatment options in unresectable neuroendocrine neoplasms (NEN) [9]. Grading can be based on H\&E sections by counting mitotic figures in an area of $2 \mathrm{~mm}^{2}$ (10 high power fields (HPF)). In the last decade, grading using immunohistochemical staining of the proliferation marker Ki67, an antigen expressed in G1, G2, S, and M-phase of the cell cycle, has been considered to be more precise $[10,11]$. The immunohistochemical staining is performed using the monoclonal antibody MIB1 [6, 7, 12].

Grading using MIB1 labeling has indeed been shown to be a good prognostic marker in many retrospective studies [3-7, 13, 14]. According to the actual WHO 2010 classification, two approaches exist to determine tumor grade: counting 
mitosis in an area of $2 \mathrm{~mm}^{2}$ on $\mathrm{H} \& \mathrm{E}$ and counting positive cells in 2000 tumor cells using MIB1 staining. Although the latter is considered difficult to apply in routine practice, some studies suggest a better stratification by using the labeling index $[15,16]$.

The optimal method to assess the MIB1 index is topic of ongoing discussion. Counting 2000 tumor nuclei is a timeconsuming process suitable for study purposes and is considered the gold standard. In daily practice, pathologists frequently choose to estimate the proliferation index by "eyeballing" or computer-assisted automated analysis to avoid this timeconsuming procedure [15]. While some studies reported all methods equally suitable [17], important differences between the three methods were instead reported by others [18-21].

Interobserver and interlaboratory comparisons have been performed for MIB1 staining for other tumor entities as well, and evidence indicating some variability has been reported [21 22, 23]. Interlaboratory differences for MIB1 staining have been stated as of minor importance [21, 23]. However, these studies focused on highly proliferative lesions and differences in the 1 to $20 \%$ range, which are crucial for NET grading, have not been examined. Studies regarding interlaboratory differences in this low range of MIB1 index have not been performed in general and for NET more specifically.

To assess potential interlaboratory differences in NET, we performed Ki67 staining in three different university hospitalbased pathology laboratories on one well-characterized patient cohort of pancreatic NET (pNET). We did not want to assess interobserver differences, so scoring of all stained slides was performed by one person using a clearly defined approach. To assess reliability of the different stains, we performed intraclass correlation as well as log-rank analysis, considering tumor-specific survival.

Our study shows significant interlaboratory differences in intensity and number of MIB1-stained cells, which highlights the need for standardization.

\section{Materials and methods}

\section{Patients and tumor specimens}

We included 61 patients with well-differentiated primary pNET and available follow-up data, who underwent surgical excision (26 males, 35 women) from 1974 to 2004, as previously described in Schmitt et al. [24]. The study was approved by the local ethics committee (StV 40-2005).

Only G1 and G2 tumors were included, classified based on mitotic count according to the WHO guidelines in 2010 and ENETS guidelines 2006. Mitoses were evaluated on whole slide sections in an area of $2 \mathrm{~mm}^{2}$ (A.P., A.S.).
A tissue micro array (TMA) comprising randomly punched areas of these 61 primary tumors [24] was used for interlaboratory comparison. Punch diameter was $0.6 \mathrm{~mm}$ comprising an area of $0.28 \mathrm{~mm}^{2}$.

\section{Immunohistochemistry}

Four-micrometer sections of the tissue micro array were stained in three different university hospital-based pathology laboratories with MIB1 antibody, according to their current protocols for diagnostic staining (Table 1). A MIB1 stainingbased proliferation index was established in random tumor areas. In a first step, all tumor cell nuclei per punch were counted with a counting grid and counting device (mean 1702 , range 267 to 5520 tumor cells) followed by evaluating all positive nuclei. Tumor cells with faint and dot-like staining were considered positive as well, because the Ki67 staining pattern varies during different phases of the cell cycle $[10,11]$. Labeling index was calculated by dividing the number of positive nuclei by the total number of tumor nuclei. Immunohistochemically stained slides were evaluated blinded to the mitotic count and clinicopathological data. Examples of immunohistochemical staining are given in Fig. 1.

\section{Statistical analysis}

Time interval from surgical intervention to tumor-induced death (TTD) was assessed by log-rank test, using GraphPad Prism4 software (GraphPad Software, Inc., San Diego, CA). $p$ values $<0.05$ were considered to indicate statistical significance. Linear regression analysis was performed with SPSS version 16.0.1 (SPSS ${ }^{\circledR}$ software, Chicago, IL, USA). Intraclass correlation was performed with SAS version 9.2 (SAS institute, Cary, NC, USA).

\section{Results}

\section{Patients and tumor specimens}

Patient age ranged from 14 to 83 years (mean 56.4 years; median 56 years). Male to female ratio was $0.74: 1$. Followup ranged from 1 to 323 months (mean 106.3 months; median 86 months). Tumor size ranged from 0.4 to $15 \mathrm{~cm}$ with (mean diameter $3.7 \mathrm{~cm}$; median diameter $3.0 \mathrm{~cm}$ ). Tumor relapse occurred in $24 / 61$ patients (39\%; mean time interval 93.9 months, median 72.5 months). Of the patients, 14/ $61(23 \%)$ died of tumor (mean time interval 106.3 months; median 85.5 months). Lymph node metastases were found in 15/61 patients (25\%); 46/61 patients had no lymph node metastases documented at time of surgery $(75 \%)$. Distant metastases occurred in $9 / 61$ patients $(15 \%)$; 52/61 patients 
Table 1 Comparison of MIB1 staining conditions for institutes 1, 2, and 3

\begin{tabular}{|c|c|c|c|c|}
\hline Institute & Antibody/clone & Pretreatment/incubation time & Dilution & Detection system \\
\hline 1 & MIB1, Dako & $\begin{array}{l}\mathrm{CC} 1, \mathrm{pH} 8.4 \\
\text { Heat-pretreatment, } 30 \mathrm{~min}\end{array}$ & $1: 20$ & Ventana BenchMark \\
\hline 2 & MIB1, Immunotech S.A. & $\begin{array}{l}\text { EDTA, pH } 8.8 \\
\text { Heat-pretreatment, } 20 \text { min }\end{array}$ & $1: 50$ & Dako Envision \\
\hline 3 & MIB1, Dako & $\begin{array}{l}\text { Tris/EDTA-buffer, } \mathrm{pH} 9.0 \\
\text { Heat-pretreatment, } 30 \mathrm{~min}\end{array}$ & $1: 100$ & Dako Real Detection system \\
\hline
\end{tabular}

Fig. 1 Example of two tumor cores with MIB1 staining of each institute: patient aP129 important differences, institute 1 (a),

institute 2 (b), and institute 3 (c); patient aP081 minor differences, institute $1(\mathbf{d})$, institute $2(\mathbf{e})$, and institute 3 (f)

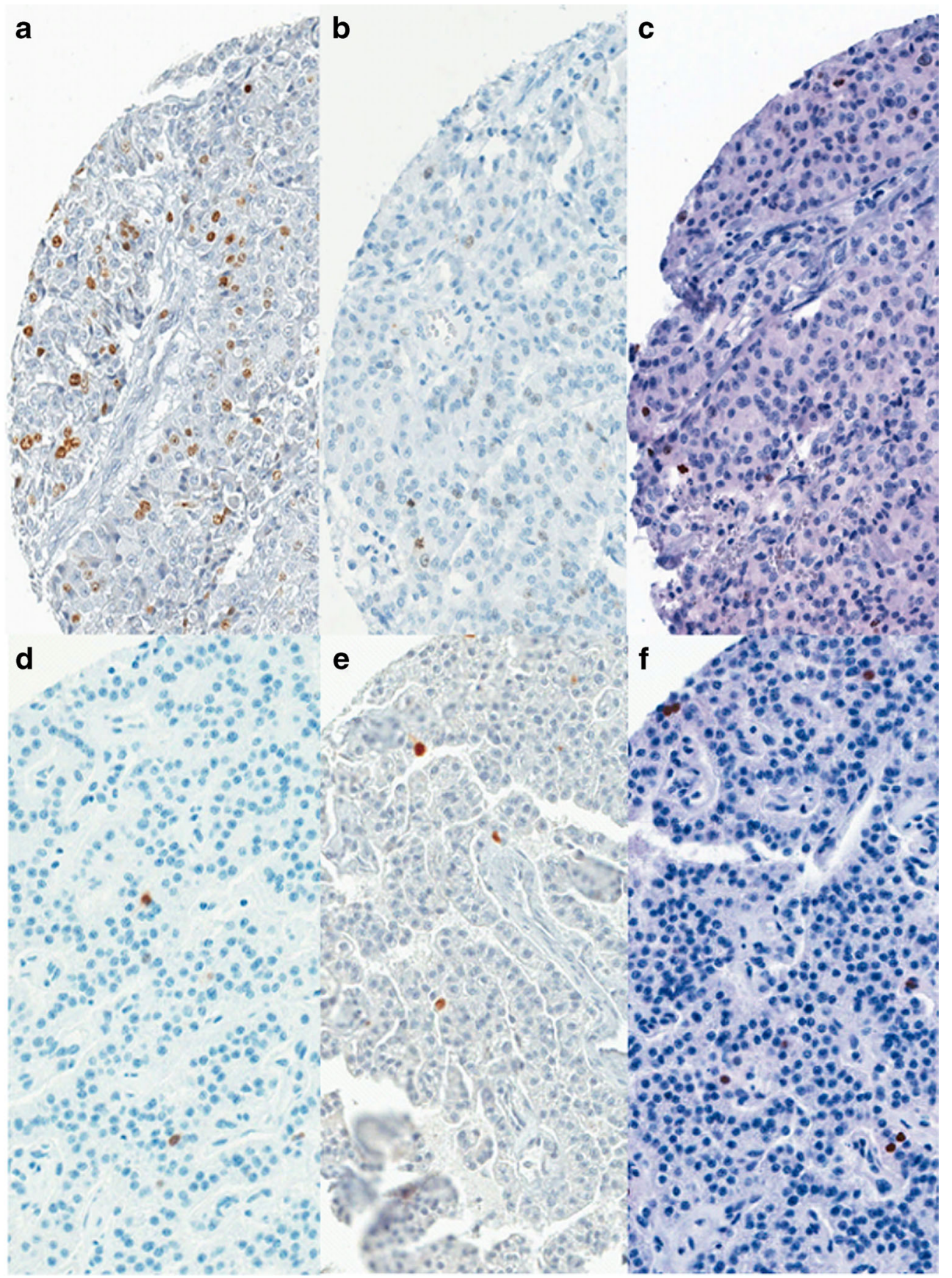


had no distant metastases documented at time of surgery (85\%) (Table 2).

\section{MIB1 staining-based proliferation index}

Significant differences in intensity as well as proportion of positive tumor nuclei were seen, even at low magnification. Highest staining intensity was provided by institute 1 , whereas that from institute 3 was weakest. Log-rank analysis for calculated proliferation indices rounded to $0.5 \%$ was performed. Chi-square values and $p$ values were recorded. The highest chi-square value correlated with the lowest $p$ value and indicated the best cutoff value for the separation between G1 and G2 tumors [25]. Regarding survival, a prognostic cutoff of $5.0 \%$ was found for institute $1,3.0 \%$ for institute 2 , and $0.5 \%$ for institute 3 for the differentiation between G1 and G2 tumors (Fig. 2a-c).

On slides stained in institute 1 , survival of patients with a proliferation index $>5.0 \%$ was significantly poorer $(p<0.001)$. A cutoff of $3.0 \%$ showed a significant difference in survival but with lower significance $(p=0.001)$, whereas a cutoff of $0.5 \%$ did not show a significant difference in survival $(p=0.361)$.

On slides stained in institute 2 , survival of patients with a proliferation index $>3.0 \%$ was significantly poorer $(p<0.001)$. A cutoff of $5.0 \%$ showed the same significant difference $(p<0.001)$; a cutoff of $0.5 \%$ also showed a significant difference.

On slides stained in institute 3 , survival of patients with a proliferation index $>0.5 \%$ was significantly poorer $(p=<0.001)$. A cutoff of 5.0 and $3.0 \%$ showed no significant difference in survival ( $p=0.800$ and 0.198 ) (Table 3).

Linear regression analysis showed a significant positive correlation between mitotic counts and MIB1 staining-based proliferation index for institutes 1 and $2\left(p=0.000, R^{2}=0.383\right.$ and $p=0.000, R^{2}=0.416$ ), whereas no significant correlation was found for institute $3\left(p=0.260, R^{2}=0.033\right)$ (Fig. 3a-c).

Strong positive correlation was found between MIB1 staining-based proliferation index from institutes 1 and 2 ( $\left.p=0.000, R^{2}=0.358\right)$ (Fig. 3d). Borderline significance was found between institutes 1 and $3\left(p=0.049, R^{2}=0.93\right)$, and no correlation was found between institutes 2 and 3 $\left(p=0.508, R^{2}=0.11\right)$ (data not shown).

Intraclass correlation found a correlation coefficient of 0.51 for the proliferation index from institutes 1 and 2 and a correlation coefficient of 0.32 for institutes 1,2 , and 3 .

\section{Grading shifts}

To differentiate between G1 and G2 tumors, a cutoff of $2.0 \%$ was applied to the proliferation indices. For slides stained in institute 1, this resulted in $50 \mathrm{G} 1$ and $11 \mathrm{G} 2$ tumors, whereas
Table 2 Clinical characteristics of 61 patients with pancreatic neuroendocrine tumors; TNMclassification according to Rindi et al. [1]

\begin{tabular}{llll}
\hline TMA & Total & Men & Women \\
\hline Patients & $61(100 \%)$ & $26(42.6 \%)$ & $35(57.4 \%)$ \\
$\quad$ Tumor-related death & $14(23.0 \%)$ & $8(13.1 \%)$ & $6(9.8 \%)$ \\
$\quad$ No tumor-related death/alive & $47(77.0 \%)$ & $18(29.5 \%)$ & $29(47.5 \%)$ \\
Follow-up & & & \\
Range (month) & $1-323$ & $3-285$ & $1-323$ \\
Mean (month) & 106.3 & 86.08 & 121.31 \\
Median (month) & 85.5 & 73 & 107 \\
T stage & & & $11(18.0 \%)$ \\
T1 & $16(26.2 \%)$ & $5(8.2 \%)$ & $13(21.3 \%)$ \\
T2 & $24(39.3 \%)$ & $11(18.0 \%)$ & $9(14.8 \%)$ \\
T3 & $14(23.0 \%)$ & $5(8.2 \%)$ & 0 \\
T4 & $1(1.6 \%)$ & $1(1.6 \%)$ & $2(3.3 \%)$ \\
Unknown & $6(9.8 \%)$ & $4(6.6 \%)$ & $10(16.4 \%)$ \\
N stage & & & $5(8.2 \%)$ \\
N0 & $14(23.0 \%)$ & $4(6.6 \%)$ & $20(32.8 \%)$ \\
N1 & $15(24.6 \%)$ & $10(16.4 \%)$ & $29(47.5 \%)$ \\
Unknown & $32(52.5 \%)$ & $12(19.7 \%)$ & $4(6.6 \%)$ \\
Mitoses per 2 mm ${ }^{2}$ & $4(6.6 \%)$ & $17(27.9 \%)$ & 0 \\
$<2$ & $46(75.4 \%)$ & 0 & $2(3.3 \%)$ \\
$2-20$ & $11(18.0 \%)$ & & \\
$>20$ & 0 & & \\
Unknown & & & \\
\hline
\end{tabular}




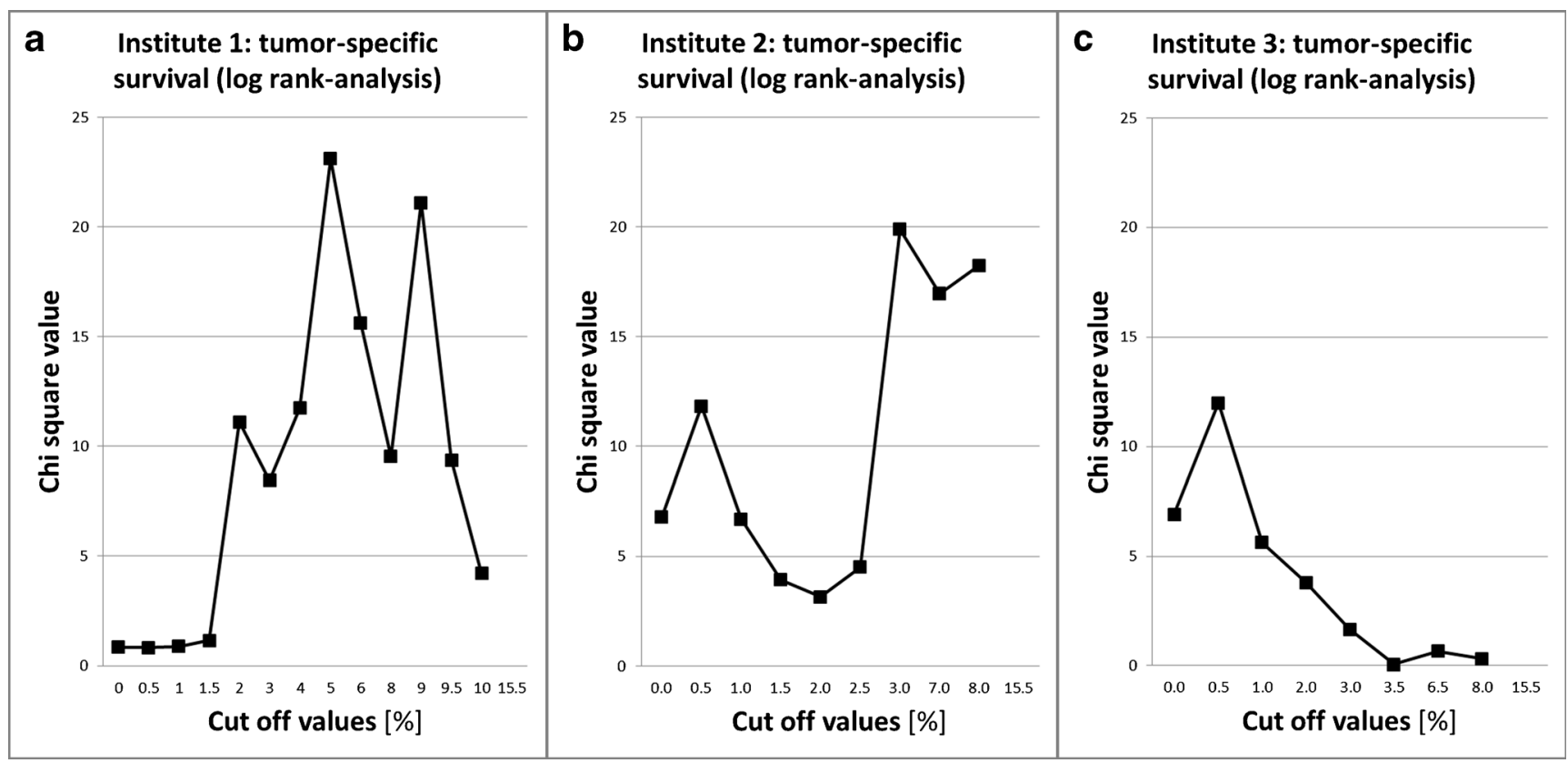

Fig. 2 Chi-square values for rounded proliferation indices: the higher the chi-square value, the better the stratification between G1 and G2 according to survival; staining institute 1 (a), staining institute 2 (b), and staining institute 3 (c)

for slides stained in institute 2, this was $53 \mathrm{G} 1$ and $8 \mathrm{G} 2$ tumors. Grading shifts were observed in nine cases $(15 \%)$. Even though staining in institute 1 was generally stronger, grade shifts went in both directions: three tumors shifted from G1 to $\mathrm{G} 2$ on slides stained in institute 1, while six tumors shifted from $\mathrm{G} 2$ to G1. Because of missing correlation between immunohistochemical MIB1 staining and mitotic count in institute 3 , we considered this staining protocol as unreliable and performed no analysis of grading shifts for this institute.

\section{Discussion}

Tumor grade and stage are to date the most reliable factors used in clinical practice to predict prognosis of pNET [2 7]

Table 3 Prognostic value of different cutoffs, estimated by log-rank analysis

\begin{tabular}{llll}
\hline & Staining institute 1 & Staining institute 2 & Staining institute 3 \\
\hline$\leq 5.0 \%$ & $55 / 61(90.2 \%)$ & $58 / 61(95.1 \%)$ & $39 / 42(92.9 \%)$ \\
$>5.0 \%$ & $6 / 61(9.8 \%)$ & $3 / 61(4.9 \%)$ & $3 / 42(7.1 \%)$ \\
$p$ value & $<0.001$ & $<0.001$ & 0.800 \\
$\leq 3.0 \%$ & $51 / 61(83.6 \%)$ & $58 / 61(95.1 \%)$ & $38 / 42(90.5 \%)$ \\
$>3.0 \%$ & $10 / 61(16.4 \%)$ & $3 / 61(4.9 \%)$ & $4 / 42(9.5 \%)$ \\
$p$ value & 0.001 & $<0.001$ & 0.198 \\
$\leq 0.5 \%$ & $25 / 61(41.0 \%)$ & $37 / 61(60.7 \%)$ & $30 / 42(71.4 \%)$ \\
$>0.5 \%$ & $36 / 61(59.0 \%)$ & $24 / 61(39.3 \%)$ & $12 / 42(28.6 \%)$ \\
$p$ value & 0.361 & 0.001 & $<0.001$ \\
\hline
\end{tabular}

and are supported by WHO and ENETS [6 26, 27]. Intriguingly, not much attention has been paid to potential interlaboratory differences, even though for the analysis of parameters such as chemical (electrolytes) and biological variables (e.g., Chromogranin-A measurement in the serum), each laboratory needs to indicate local reference values [28, 29]. For MIB1 staining, the antibody batch used will be different and different laboratories use different staining protocols. In addition, pre-analytical variables such as transport times, fixation times, and tissue processing procedures are not standardized. Few data exist regarding interlaboratory variability of Ki67 proliferation index [21], and only one study from 2002 reported this, among other tumors, in pNET [23].

To assess the extent of interlaboratory Ki67 staining variability, we assessed staining of 61 tumors on TMA slides stained in three different university hospital-based pathology laboratories, all regularly participating in quality assurance assessments with good results. We found significant and relevant differences in staining intensity and proportion of MIB1 positive cells in immunohistochemically stained slides impacting on the proliferation index, notably in low proliferating tumors. This calls for external standardization.

Because of missing correlation between immunohistochemical MIB1 staining and mitotic count in institute 3, we considered this staining protocol as unreliable. We therefore focused statistical analysis on a comparison of institutes 1 and 2. In fact, on stained slides from both institutes, clear prognostic stratification could be attained. Considering staining results from these two institutes, a shift in grade was observed in nine cases, accounting for $15 \%$ of tumors. Proliferation indices from both correlated well with mitotic counts. Using 
a

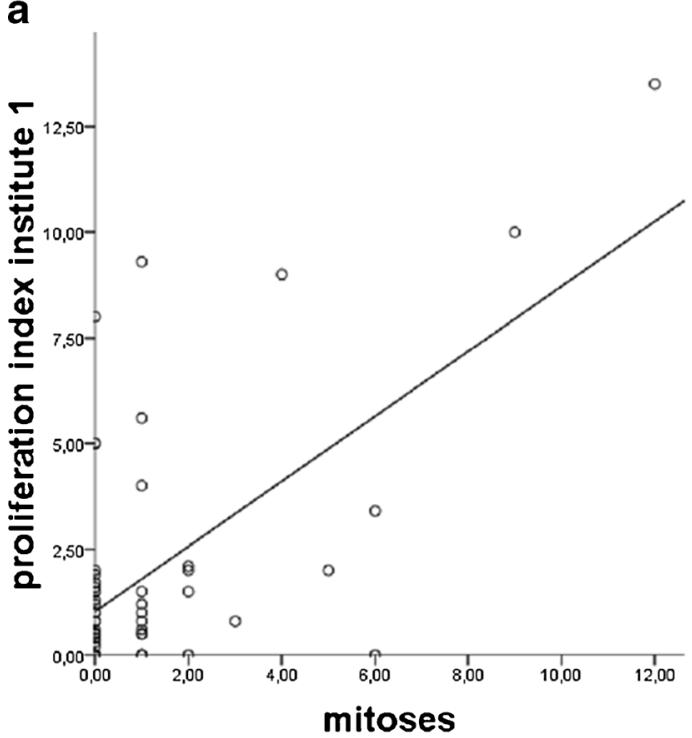

C

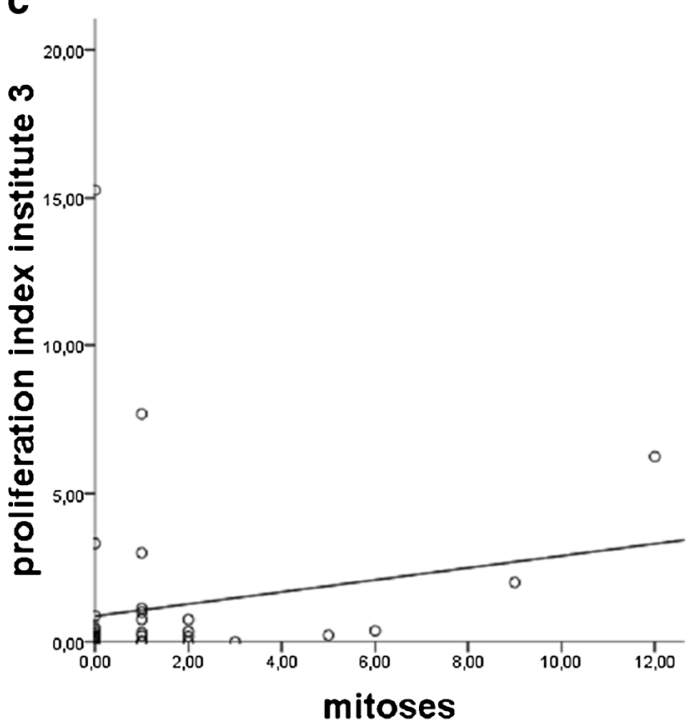

Fig. 3 Regression analysis: proliferation index (institute 1) and mitoses, $p=0.000, R^{2}=0.383(\mathbf{a})$; proliferation index (institute 2 ) and mitoses, $p=0.000, R^{2}=0.416(\mathbf{b})$; proliferation index (institute 3 ) and mitoses,

log-rank analysis, an optimal cutoff of $5.0 \%$ was established for institute 1 and of $3.0 \%$ for institute 2 . As we used a TMA composed of randomly chosen tumor areas, these cutoff values are expected to be different from those in studies based upon counting in hotspots. Our cutoff values can therefore not be taken as generally applicable. A random area rather than a hotspot approach has been shown to result in lower proliferation indices [30].

A potential limiting factor of our study is the use of consecutive sections, a few micrometers apart, which could account for slight differences. This however is compensated by the relatively large number of tumors b

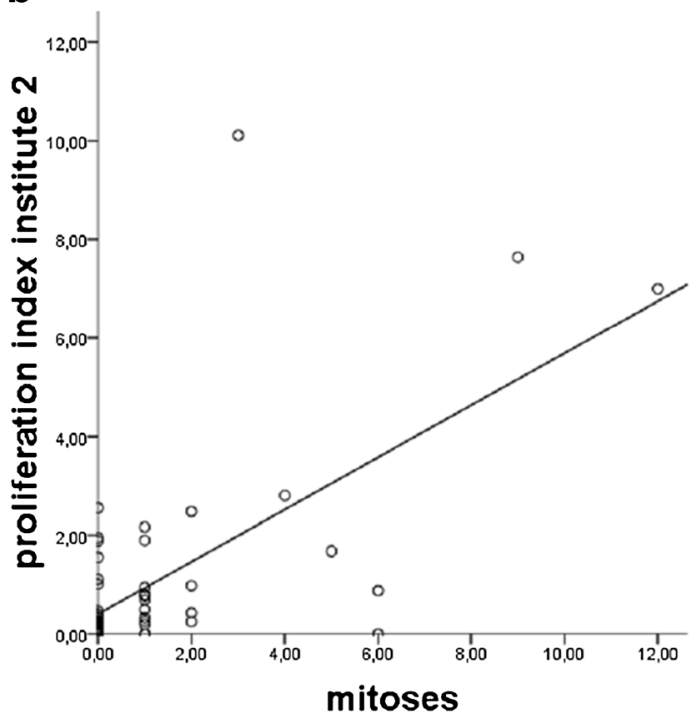

O Observed
Linear

\section{d}

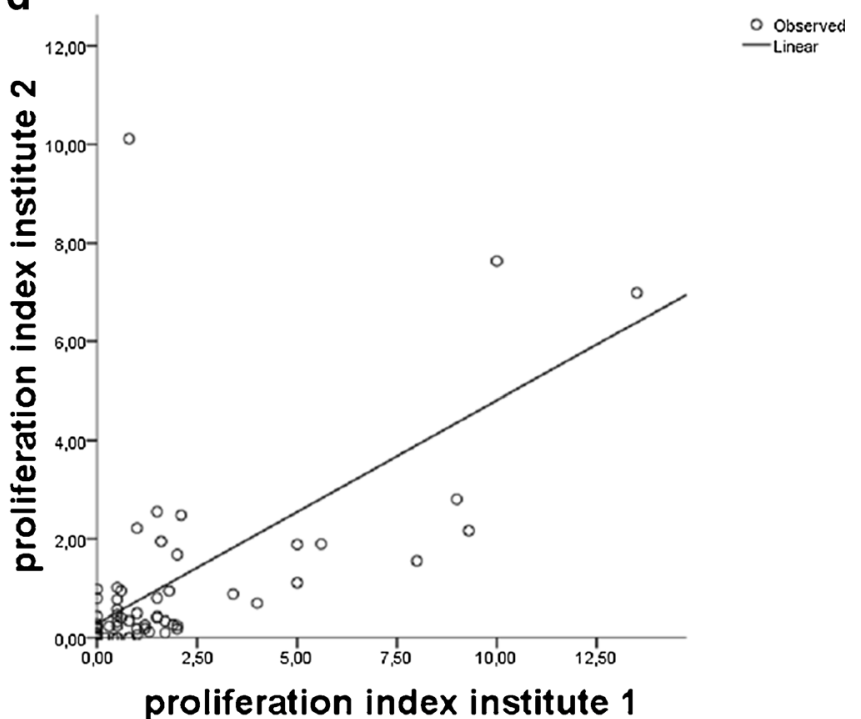

$p=0.260, R^{2}=0.033(\mathbf{c})$, proliferation index institutes 1 and $2, p=0.000$, $R^{2}=0.358(\mathbf{d})$

included in the study. We conclude that the observed interlaboratory differences are a result of variations in staining procedures between the participating laboratories. Our findings are concordant with other investigations regarding interlaboratory variability, which emphasized the role of processing and fixation processes [31-33], different reagents and pretreatment for immunohistochemical staining [34]; [35, 36], while antibody dilution was found to be less important [37] [23].

Our results indicate that differences in MIB1 stainingbased proliferation indices are likely to be higher in tumors with low proliferative activity. This finding is of importance in 
NET because the cutoff values for G1 and G2 tumors are in a very low proliferation range. This must be accounted for using potential external calibrators and must be checked in quality control measures.

We used log-rank analysis to define a cutoff value for the proliferation index based upon the slides stained in each of the three institutes. The cutoff value obtained for each institute appropriately stratified the patient cohort on the stained slide set on which it was calculated but was less significant when applied to the slides stained in one of the other institutes. This result might explain why in the literature different cutoff values have been published $[2,7,24]$. While significant interlaboratory differences were found, all had prognostic value, which underlines the prognostic importance of Ki67 staining-based proliferation index as has been repeatedly reported [3-7, 13, 14]. Our results, however, also indicate that minimizing interlaboratory variability is a priority. Grading based on proliferative activity may impact on therapeutic decisions, and consistent and reproducible grading is mandatory to maintain comparability of clinical studies. Two approaches might be considered to attain this goal. The first is standardization of staining intensity but to this end, an external benchmark needs to be artificially defined. This could be the immunohistochemical staining result obtained in a reference institute, allowing application of a "correction factor" for other institutes based on interlaboratory comparison. Alternatively, an external calibrator could be introduced such as a slow growing cell line, of which proliferative activity can be defined using BrDU incorporation over a defined timespan. Whichever the chosen solution, intralaboratory reproducibility has to be monitored by regular quality control measures such as round robin tests.

Our data contribute to the ongoing discussion on the optimal approach to establish proliferative activity, to the definition of the cutoff value to be used for NET grading [3-7, 13, 14, 16] and to the need for standardization of MIB1 staining. Since proliferation indexbased grades impact on therapy decisions and followup intervals [14], reliability and reproducibility of their assessment are essential. This is not limited to pNET but holds true for a variety of tumors including breast cancer [21, 38], melanoma [39], and bronchopulmonary carcinoids [40, 41].

Conflict of interests The authors declare that they have no competing interests.

Funding This work was partially supported by the Swiss National Science Foundation (Grant No. 310030_144236 to Aurel Perren).

\section{References}

1. Couvelard A, O'Toole D, Turley H, Leek R, Sauvanet A, Degott C, Ruszniewski P, Belghiti J, Harris AL, Gatter K, Pezzella F (2005) Microvascular density and hypoxia-inducible factor pathway in pancreatic endocrine tumours: negative correlation of microvascular density and VEGF expression with tumour progression. $\mathrm{Br} \mathrm{J}$ Cancer 92:94-101. doi:10.1038/sj.bjc.6602245

2. Hochwald SN, Zee S, Conlon KC, Colleoni R, Louie O, Brennan MF, Klimstra DS (2002) Prognostic factors in pancreatic endocrine neoplasms: an analysis of 136 cases with a proposal for low-grade and intermediate-grade groups. J Clin Oncol 20:2633-2642

3. La Rosa S, Sessa F, Capella C, Riva C, Leone BE, Klersy C, Rindi G, Solcia E (1996) Prognostic criteria in nonfunctioning pancreatic endocrine tumours. Virchows Arch 429:323-333

4. Pelosi G, Bresaola E, Bogina G, Pasini F, Rodella S, Castelli P, Iacono C, Serio G, Zamboni G (1996) Endocrine tumors of the pancreas: Ki-67 immunoreactivity on paraffin sections is an independent predictor for malignancy: a comparative study with proliferating-cell nuclear antigen and progesterone receptor protein immunostaining, mitotic index, and other clinicopathologic variables. Hum Pathol 27:1124-1134

5. Ekeblad S, Skogseid B, Dunder K, Oberg K, Eriksson B (2008) Prognostic factors and survival in 324 patients with pancreatic endocrine tumor treated at a single institution. Clin Cancer Res 14: 7798-7803. doi:10.1158/1078-0432.CCR-08-0734

6. Rindi G, Kloppel G, Alhman H, Caplin M, Couvelard A, de Herder WW, Erikssson B, Falchetti A, Falconi M, Komminoth P, Korner M, Lopes JM, McNicol AM, Nilsson O, Perren A, Scarpa A, Scoazec JY, Wiedenmann B (2006) TNM staging of foregut (neuro)endocrine tumors: a consensus proposal including a grading system. Virchows Arch 449:395-401

7. Scarpa A, Mantovani W, Capelli P, Beghelli S, Boninsegna L, Bettini R, Panzuto F, Pederzoli P, Delle Fave G, Falconi M (2010) Pancreatic endocrine tumors: improved TNM staging and histopathological grading permit a clinically efficient prognostic stratification of patients. Mod Pathol 23:824-833. doi:10.1038/ modpathol.2010.58

8. Heitz P, Komminoth P, Perren A, Klimstra DS, Dayal Y, Bordi C, Lechago J, Centeno BA, Klöppel G (2004) WHO classification of tumours, Pathology and genetics of tumours of endocrine organs. In: De Lellis R, Heitz P, Lloyd R, Eng C (eds) Tumours of the endocrine pancreas (chapter 4). IARC, Lyon

9. Pavel M, Baudin E, Couvelard A, Krenning E, Oberg K, Steinmuller T, Anlauf M, Wiedenmann B, Salazar R, Conference BC (2012) ENETS consensus guidelines for the management of patients with liver and other distant metastases from neuroendocrine neoplasms of foregut, midgut, hindgut, and unknown primary. Neuroendocrinology 95:157-176. doi:10.1159/000335597

10. Cattoretti G, Becker MH, Key G, Duchrow M, Schluter C, Galle J, Gerdes J (1992) Monoclonal antibodies against recombinant parts of the Ki-67 antigen (MIB 1 and MIB 3) detect proliferating cells in microwave-processed formalin-fixed paraffin sections. J Pathol 168:357-363. doi:10.1002/path.1711680404

11. Gerdes J, Schwab U, Lemke H, Stein H (1983) Production of a mouse monoclonal antibody reactive with a human nuclear antigen associated with cell proliferation. Int J Cancer 31:13-20

12. Jamali M, Chetty R (2008) Predicting prognosis in gastroenteropancreatic neuroendocrine tumors: an overview and the value of $\mathrm{Ki}-$ 67 immunostaining. Endocr Pathol 19:282-288

13. Clarke MR, Baker EE, Weyant RJ, Hill L, Carty SE (1997) Proliferative activity in pancreatic endocrine tumors: association with function, metastases, and survival. Endocr Pathol 8:181-187

14. Panzuto F, Boninsegna L, Fazio N, Campana D, Pia Brizzi M, Capurso G, Scarpa A, De Braud F, Dogliotti L, Tomassetti P, 
Delle Fave G, Falconi M (2011) Metastatic and locally advanced pancreatic endocrine carcinomas: analysis of factors associated with disease progression. J Clin Oncol 29:2372-2377

15. Klimstra DS, Modlin IR, Adsay NV, Chetty R, Deshpande V, Gonen M, Jensen RT, Kidd M, Kulke MH, Lloyd RV, Moran C, Moss SF, Oberg K, O'Toole D, Rindi G, Robert ME, Suster S, Tang LH, Tzen CY, Washington MK, Wiedenmann B, Yao J (2010) Pathology reporting of neuroendocrine tumors: application of the Delphic consensus process to the development of a minimum pathology data set. Am J Surg Pathol 34:300-313

16. Lowe K, Khithani A, Liu E, Winston T, Christian D, Saad J, Jeyarajah DR (2012) Ki-67 labeling: a more sensitive indicator of malignant phenotype than mitotic count or tumor size? J Surg Oncol 106:724-727

17. Rindi G, Klersy C, Inzani F, Fellegara G, Ampollini L, Ardizzoni A, Campanini N, Carbognani P, De Pas TM, Galetta D, Granone PL, Righi L, Rusca M, Spaggiari L, Tiseo M, Viale G, Volante M, Papotti M, Pelosi G (2014) Grading the neuroendocrine tumors of the lung: an evidence-based proposal. Endocr Relat Cancer 21:116. doi:10.1530/ERC-13-0246

18. Goodell PP, Krasinskas AM, Davison JM, Hartman DJ (2012) Comparison of methods for proliferative index analysis for grading pancreatic well-differentiated neuroendocrine tumors. Am J Clin Pathol 137:576-582. doi:10.1309/AJCP92UCXPJMMSDU

19. Reid MD, Bagci P, Ohike N, Saka B, Erbarut Seven I, Dursun N, Balci S, Gucer H, Jang KT, Tajiri T, Basturk O, Kong SY, Goodman M, Akkas G, Adsay V (2015) Calculation of the Ki67 index in pancreatic neuroendocrine tumors: a comparative analysis of four counting methodologies. Mod Pathol 28:686-694. doi:10.1038/ modpathol.2014.156

20. Tang LH, Gonen M, Hedvat C, Modlin IM, Klimstra DS (2012) Objective quantification of the Ki67 proliferative index in neuroendocrine tumors of the gastroenteropancreatic system: a comparison of digital image analysis with manual methods. Am J Surg Pathol 36:1761-1770. doi:10.1097/PAS.0b013e318263207c

21. Varga Z, Diebold J, Dommann-Scherrer C, Frick H, Kaup D, Noske A, Obermann E, Ohlschlegel C, Padberg B, Rakozy C, Sancho Oliver S, Schobinger-Clement S, Schreiber-Facklam H, Singer G, Tapia C, Wagner U, Mastropasqua MG, Viale G, Lehr HA (2012) How reliable is Ki-67 immunohistochemistry in grade 2 breast carcinomas? A QA study of the Swiss Working Group of Breast- and Gynecopathologists. PLoS One 7:e37379. doi:10.1371/journal. pone. 0037379

22. Hsu CY, Ho DM, Yang CF, Chiang H (2003) Interobserver reproducibility of MIB-1 labeling index in astrocytic tumors using different counting methods. Mod Pathol 16:951-957. doi:10.1097/01. MP.0000084631.64279.BC

23. Mengel M, von Wasielewski R, Wiese B, Rudiger T, MullerHermelink HK, Kreipe H (2002) Inter-laboratory and interobserver reproducibility of immunohistochemical assessment of the Ki-67 labelling index in a large multi-centre trial. J Pathol 198:292-299

24. Schmitt AM, Anlauf M, Rousson V, Schmid S, Kofler A, Riniker F, Bauersfeld J, Barghorn A, Probst-Hensch NM, Moch H, Heitz PU, Kloeppel G, Komminoth P, Perren A (2007) WHO 2004 criteria and CK19 are reliable prognostic markers in pancreatic endocrine tumors. Am J Surg Pathol 31:1677-1682. doi:10.1097/PAS. 0b013e31805f675d

25. Voss SM, Riley MP, Lokhandwala PM, Wang M, Yang Z (2015) Mitotic count by phosphohistone H3 immunohistochemical staining predicts survival and improves interobserver reproducibility in well-differentiated neuroendocrine tumors of the pancreas. Am J Surg Pathol 39:13-24. doi:10.1097/PAS.0000000000000341
26. Klimstra DSCC, Arnold R (2010) Neuroendocrine neoplasms of the pancreas. IARC, Lyon

27. Kloppel G, Perren A, Heitz PU (2004) The gastroenteropancreatic neuroendocrine cell system and its tumors: the WHO classification. Ann N Y Acad Sci 1014:13-27

28. Muller MM (2000) Implementation of reference systems in laboratory medicine. Clin Chem 46:1907-1909

29. Verderio P, Dittadi R, Marubini E, Pizzamiglio S, Gion M, De Apollonia L, Paradiso A, Italian Network for Quality Assessment of Tumor Biomarkers Group (2007) An Italian program of external quality control for chromogranin $\mathrm{A}(\mathrm{CgA})$ assay: performance evaluation of CgA determination. Clin Chem Lab Med 45:1244-1250. doi:10.1515/CCLM.2007.251

30. Yang Z, Tang LH, Klimstra DS (2011) Effect of tumor heterogeneity on the assessment of Ki67 labeling index in well-differentiated neuroendocrine tumors metastatic to the liver: implications for prognostic stratification. Am J Surg Pathol 35:853-860. doi:10. 1097/PAS.0b013e31821a0696

31. Bai Y, Tolles J, Cheng H, Siddiqui S, Gopinath A, Pectasides E, Camp RL, Rimm DL, Molinaro AM (2011) Quantitative assessment shows loss of antigenic epitopes as a function of pre-analytic variables. Lab Investig 91:1253-1261. doi:10.1038/labinvest.2011. 75

32. Benini E, Rao S, Daidone MG, Pilotti S, Silvestrini R (1997) Immunoreactivity to MIB-1 in breast cancer: methodological assessment and comparison with other proliferation indices. Cell Prolif 30:107-115

33. Srinivasan M, Sedmak D, Jewell S (2002) Effect of fixatives and tissue processing on the content and integrity of nucleic acids. Am J Pathol 161:1961-1971. doi:10.1016/S0002-9440(10)64472-0

34. Frost AR, Sparks D, Grizzle WE (2000) Methods of antigen recovery vary in their usefulness in unmasking specific antigens in immunohistochemistry. Appl Immunohistochem Mol Morphol 8: 236-243

35. Heuschmid M, Hofmockel G, Dexler B, Dammrich J, Bassukas ID (2002) Different antigen unmasking techniques lead to significant differences in immunohistochemical staining of Ki-67 (Mib-1) in renal cell carcinomas. Oncol Rep 9:19-22

36. Munakata S, Hendricks JB (1993) Effect of fixation time and microwave oven heating time on retrieval of the $\mathrm{Ki}-67$ antigen from paraffin-embedded tissue. J Histochem Cytochem 41:1241-1246

37. McCormick D, Yu C, Hobbs C, Hall PA (1993) The relevance of antibody concentration to the immunohistological quantification of cell proliferation-associated antigens. Histopathology 22:543-547

38. Meyer JS, Alvarez C, Milikowski C, Olson N, Russo I, Russo J, Glass A, Zehnbauer BA, Lister K, Parwaresch R, Cooperative Breast Cancer Tissue Resource (2005) Breast carcinoma malignancy grading by Bloom-Richardson system vs proliferation index: reproducibility of grade and advantages of proliferation index. Mod Pathol 18:1067-1078. doi:10.1038/modpathol.3800388

39. Vereecken P, Laporte M, Heenen M (2007) Significance of cell kinetic parameters in the prognosis of malignant melanoma: a review. J Cutan Pathol 34:139-145. doi:10.1111/j.1600-0560.2006. 00588.x

40. Grimaldi F, Muser D, Beltrami CA, Machin P, Morelli A, Pizzolitto S, Talmassons G, Marciello F, Colao AA, Monaco R, Monaco G, Faggiano A (2011) Partitioning of bronchopulmonary carcinoids in two different prognostic categories by ki-67 score. Front Endocrinol 2:20. doi:10.3389/fendo.2011.00020

41. Zahel T, Krysa S, Herpel E, Stenzinger A, Goeppert B, Schirmacher P, Hoffmann H, Schnabel PA, Warth A (2012) Phenotyping of pulmonary carcinoids and a Ki-67-based grading approach. Virchows Arch 460:299-308 